\title{
Incomplete Information based Collaborative Computing in Emergency Communication Networks
}

\author{
Qianqian Wang, Yongxu Zhu, Member, IEEE and Xianbin Wang, Fellow, IEEE
}

\begin{abstract}
Due to the urgent and unpredictable nature of disaster relief, emergency management systems (EMS) faces an enormous challenge of real-time data analysis without the complete information from emergency communication networks (ECNs). In this letter, we propose an incomplete information based twotier game model (IITG) to realize collaborative computing at the edge of ECNs, which incentivizes idle computing devices (ICDs) to share computation resources through maximizing utilities of EMS and ICDs. Furthermore, we develop a near-optimal IITG algorithm (N-IITG) to seek the unique Bayesian Nash equilibrium. Simulation results reveal that N-IITG outperforms the existing incomplete information based methods in terms of computation latency and participants utilities.
\end{abstract}

Index Terms - collaborative computing, game theory, incomplete information, emergency communication networks

\section{INTRODUCTION}

To reduce the harmful effects of unpredictable disasters, the emergency management system (EMS) requires timely analysis of the massive amount of data for providing effective disaster relief strategies [1]. This stringent requirement brings a significant challenge to real-time data computation in emergency communication networks (ECNs). Moreover, due to the highly uncertain nature of disasters, it is extremely difficult to obtain complete information of ECNs in advance [2]. Therefore, how to design an effective data processing mechanism based on incomplete information becomes essential for ECNs.

To relieve the computation pressure in ECNs, some researchers propose the collaborative computing at the edge of networks, which utilizes the spare computational resources from smartphones, desktop PCs, or other computational devices in proximity to achieve a dynamic increase in computational capacity [3], [4]. While the concept of collaborative computing is promising, engaging idle computing devices (ICDs) for sharing could be difficult as they have no commitments to do so. They may expect compensation

Manuscript received ; revised ; accepted. Date of publication ; date of current version. This work was supported by the National Natural Science Foundation of China (No. 61871446). The associate editor coordinating the review of this paper and approving it for publication was . (Corresponding author: Yongxu Zhu.)

Q. Wang is with Jiangsu Key Laboratory of Wireless Communications, Nanjing University of Posts \& Telecommunications, Nanjing 210003, China, and School of Software Engineering, Jinling Institute of Technology, Nanjing 211169, China (e-mail: 2016010202@njupt.edu.cn).

Y. Zhu is with Division of Computer Science and Informatics, London South Bank University, London SE10AA, UK (e-mail: yongxu.zhu@1sbu.ac.uk).

$\mathrm{X}$. Wang is with Department of Electrical and Computer Engineering, Western University, London, ON N6A5B9, Canada (e-mail: xianbin.wang@uwo.ca).

Identify applicable funding agency here. If none, delete this. since computation offloading potentially affects local computing tasks. Therefore, successful exploitation of collaborative computing at the edge of ECNs requires a careful design of the incentive mechanism. The game theory-based approach is one of the suitable tools to model and analyze the incentive mechanism [5]. Nevertheless, the existing mechanisms in the literature have overlooked several critical issues. First, recent literature is largely based on the assumption that the competitive participants have the full knowledge of the network and local constraints [6], [7], such as the path loss fading and computation capacities. However, it is extremely difficult to acquire such information of ECNs in advance in most realistic scenarios. Furthermore, the self-interested participants are non-cooperative in ECNs, and thus the competitions, in this case, exist not only between the EMS (service consumer) and the ICDs (service provider), but also among the ICDs. Although the previous game-theoretic studies can cope with the competition between the service provider and the service consumer [6], [8], [9] or inside service providers [7], [10], few consider the above competitive relationships simultaneously.

In this work, we propose an incomplete information based two-tier game model (IITG) to incentivize collaborative computing at the edge of ECNs, where the objective is to maximize the utilities of the EMS and the ICDs through optimizing the pricing strategy and the computing resources allocation simultaneously. Specifically, the interactions between the EMS and the ICDs are formulated as a two-tier game model, which jointly combines the Stackelberg game and the Cournot game. Through this model, the EMS can dynamically optimize its pricing mechanism, and the ICDs can select the optimal computation workload accordingly. Furthermore, depending on what the EMS and the ICDs know, we seek the Bayesian Nash equilibrium (BNE) under incomplete information, and a near-optimal IITG (N-IITG) algorithm is developed to reach the unique BNE by iterations. Simulation results demonstrate that our design achieves a near-optimal performance of complete information and outperforms the existing incomplete information based methods in terms of computation latency and participants utilities.

\section{SYSTEM MODEL AND PROBLEM FORMULATION}

We consider a collaborative computing scenario illustrated in Fig. 1. There are $I$ ICDs, denoted as $\mathcal{I} \triangleq\{1,2, \ldots, I\}$, locating at the edge of ECNs to offer computational services for the EMS. During a time slot, $Q_{\mathrm{EMS}}$ bits of data are processed for emergency management, and we assume $P_{\mathrm{EMS}}$ as the unit profit that the EMS benefits from the data analysis. 
As a centralized datacenter, the EMS needs to determine the optimal unit price $p^{\text {opt }}$ (per bit) to incentivize collaborative computing. Then, the optimal amount of computing resources $q_{i}^{\text {opt }}$ is provided by each $\mathrm{ICD}_{i}$. Once the collaboration is set up, the processed data will be transmitted back to the EMS for emergency management, and each ICD gets its compensation for sharing its computation resources.

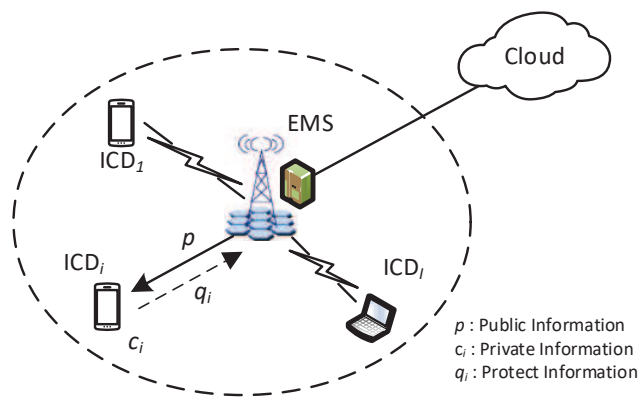

Fig. 1. Illustration of the collaborative computing architecture.

To incentivize the collaborative computing from the ICDs and satisfy the computation requirement from the EMS, the objective of this work is to jointly optimize the pricing strategy $p^{\text {opt }}$ and the computing resources allocation $\left\{\left.q_{i}^{\text {opt }}\right|_{i \in \mathcal{I}}\right\}$ to maximize the utilities of the EMS and the ICDs. We adopt the payoff to each participant as its utility. The mathematical model is described in detail as follows.

\section{A. Utility Model}

The utility of the EMS is defined as the profit through computing offloading minus the sum of its payments to the ICDs, which can be given by

$$
U_{\mathrm{EMS}}(p)=\left(P_{\mathrm{EMS}}-p\right) \min \left\{Q_{\mathrm{EMS}}, Q_{\mathrm{ICD}}\right\},
$$

where the unit profit $P_{\text {EMS }}$ benefiting from the data analysis is a known parameter, which may vary with the importance of the emergency management task. The price strategy $p$ denotes the unit price of computing offloading offered by the EMS. The total amount of the shared computing resources paid by the EMS is the minimum value of $Q_{\mathrm{EMS}}$ and $Q_{\mathrm{ICD}}$, where $Q_{\mathrm{ICD}}$ is the total amount of computing resources shared by the ICDs. We assume rationality in this work that the EMS will not buy the computing resources beyond its requirement, and the ICDs will not share computing resources without benefit.

The utility $U_{i}$ of $\mathrm{ICD}_{i}$ is defined as the reward paid by the EMS minus the cost to accomplish $q_{i}$ bits of data processing, which can be expressed as

$$
U_{i}\left(q_{i}\right)=\left(p-c_{i}\right) q_{i}
$$

where $q_{i}$ denotes the number of computing resources shared by $\mathrm{ICD}_{i}$. The unit cost $c_{i}$ (per bit) for sharing denotes the transmission energy and computing cost for offloading one bit of data, which dynamically varies with CPU performance, path loss fading, and other network environments [4].

\section{B. Incomplete information model}

Since ECNs have the characteristic of high uncertainty, it is impractical to get complete information in advance during collaborative computing. Similar to other incomplete information scenarios [8]-[10], the EMS may know each ICD's characteristics or not, such as collaboration costs $c_{i}$. Thus, we develop an incomplete information model depending on whether the information is known by others.

- Public Information: Known by all participants.

- Protect Information: Known by part of participants.

- Private Information: Only known by participant itself.

Then, we can classify the parameters in (1) and (2) into three categories as listed in Table I.

TABLE I

THE INCOMPLETE INFORMATION MODEL

\begin{tabular}{cc}
\hline Information Category & Parameter \\
\hline Public Information & $p$ \\
\hline Protect Information & $q_{i}$ \\
\hline Private Information & $Q_{\mathrm{EMS}}, P_{\mathrm{EMS}}, c_{i}$ \\
\hline
\end{tabular}

Considering the practical situation, we assume that the unit price $p$ is common knowledge of all participants, including the EMS and the ICDs. The offloading strategy $q_{i}$ is the protect information which can be obtained by the EMS and each ICD. The private information $c_{i}$ is the actual unit cost known to each ICD but is not known by EMS or other ICDs. Also, the computing task parameters $Q_{\mathrm{EMS}}$ and $P_{\mathrm{EMS}}$ are the private information of the EMS.

By exploring (1) and (2), we notice that the EMS and the ICDs have different objectives. The EMS expects the ICDs to accomplish a more massive task with a lower unit price, and the ICDs are the opposite. Meanwhile, each noncooperative ICD competes to acquire a higher payoff by sharing a larger amount of computing resources; however, with more computing resources supplied by ICD, the EMS can damp down the unit price of computing offloading according to the theory of demand and supply. Hence, the competitions exist not only between the EMS and the ICDs but also among multiple ICDs. This motivates us to propose an incomplete information based two-tier game (IITG) model to tackle the joint optimization problem of the EMS and the ICDs.

\section{TWo-TIER GAME ANALYSIS UNDER INCOMPLETE INFORMATION}

In this section, we analyze the optimal strategies of the EMS and the ICDs by jointing a Stackelberg game and a Cournot game under incomplete information. The optimization problems for the EMS and the ICDs are conducted by the following two tiers. We use the derived results to prove the existence of BNE.

- Tier I: Players: the EMS (a single leader); Strategy: the unit price $p$; Utility: $U_{\mathrm{EMS}}(p)$ given in (1).

- Tier II: Players: the ICDs (multiple competitive followers); Strategy: the shared computing resources $\left\{\left.q_{i}\right|_{i \in \mathcal{I}}\right\}$; Utility: $\left\{\left.U_{i}\left(q_{i}\right)\right|_{i \in \mathcal{I}}\right\}$ given in (2).

We first model the interaction between the EMS and the ICDs as a Stackelberg game, i.e., the pricing strategy $p$ and the amount of computation offloading $q_{i}$. In our proposed collaborative computing model, the EMS (a single leader) has the first-mover advantage to imposes the optimal unit 
price $p^{\text {opt }}$ of computation offloading for the ICDs. Then, the ICDs (multiple followers) can divide the optimal amount of computation offloading $q_{i}^{\text {opt }}$ by following the pricing strategy announced by the EMS. Both the EMS and the ICDs aim to obtain the optimal utilities by maximizing their payoff. These characteristics align well with the Stackelberg game model. The objective function of the EMS is

$$
\begin{gathered}
\max _{p^{\mathrm{opt}}} U_{\mathrm{EMS}}\left(p \mid P_{\mathrm{EMS}}, Q_{\mathrm{EMS}}, \boldsymbol{q}\right) \\
\text { s.t. } \quad 0 \leq p^{\mathrm{opt}} \leq P_{\mathrm{EMS}},
\end{gathered}
$$

where $\boldsymbol{q}=\left\{q_{1}, q_{2}, \ldots, q_{i}, \ldots, q_{I}\right\}$ represents the computation offloading strategies of all ICDs. The derived optimal unit price $p^{\text {opt }}$ paid to the ICDs should be no more than the unit profit $P_{\mathrm{EMS}}$ that the EMS can benefit from computation offloading; otherwise, the utility of the EMS will be negative.

Then, the ICDs, acting as multiple followers in the Stackelberg game, deduce their optimal shared computing resources by following the pricing strategy announced by the EMS (leader). Moreover, the internal competitions among multiple ICDs are modeled as a Cournot game, which is one of the most popular types of games used to model the interactions among multiple strategic generators. Since each ICD's payoff depends not only on its own strategy but also on the decisions of its rivals, the optimization problem for $\mathrm{ICD}_{i}$ can be expressed as

$$
\begin{aligned}
& \max _{q_{i}^{\mathrm{opt}}} U_{i}\left(q_{i} \mid p, \boldsymbol{q}_{-i}\right), \forall i \in \mathcal{I} \\
& \text { s.t. } \quad \sum_{i \in \mathcal{I}} q_{i}^{\mathrm{opt}} \leq Q_{\mathrm{EMS}}
\end{aligned}
$$

where $\boldsymbol{q}_{-i}$ is the strategy set of the ICDs other than $\mathrm{ICD}_{i}$. $\sum_{i \in \mathcal{I}} q_{i}^{\mathrm{opt}} \leq Q_{\mathrm{EMS}}$ denotes that the total amount of computation offloading is no more than the EMS's demand because all participants in the game are rational and thus will not share computing resources without benefit.

Furthermore, since the internal competition among multiple ICDs fits the Cournot game model, and thus we estimate $p$ by the Cournot price function model to depict the competitive relationship. The unit price $p$ is a decreasing function with respect to the total amount of computation offloading. It is subject to $\frac{\partial p}{\partial Q_{\mathrm{ICD}}}<0$, where $Q_{\mathrm{ICD}}=\sum_{i \in \mathcal{I}} q_{i}$. To simplify the problem, we estimate $p$ with a universal linear model as in references [7], [10], i.e.,

$$
p=\max \left\{0, p_{\max }-\alpha \sum_{i \in \mathcal{I}} q_{i}\right\},
$$

where $p_{\max }$ is the price function intercept that denotes the maximum unit price offered by the EMS, and $\alpha$ is a nonnegative coefficient that reflects the change trend of the unit price and the total amount of shared computing resources, i.e., $\alpha=-\frac{\partial p}{\partial Q_{\mathrm{ICD}}}$.

Next, we adopt backward induction [6], [8], [9] for IITG analysis as follows.

\section{A. Tier II: the optimal computing offloading strategy}

In this tier, we optimize the computing offloading strategy for each ICD given the EMS's (leader) strategy.
Theorem 1. Each $\mathrm{ICD}_{i}$ (follower) determines the optimal amount of computation offloading $q_{i}^{\text {opt }}$ given the unit price strategy $p$ and $\alpha$ from the EMS (leader), given by

$$
q_{i}^{\mathrm{opt}}=\frac{p-c_{i}}{\alpha}
$$

where $c_{i}$ is the private information known by each ICD itself.

Proof: We substitute (5) into (2); thus, the utility of $\mathrm{ICD}_{i}$ can be given by

$$
U_{i}\left(q_{i}\right)=-\alpha q_{i}^{2}+\left(p_{\max }-\alpha \sum_{k \neq i} \widehat{q_{k}}-c_{i}\right) q_{i} .
$$

Under incomplete information model, the rivals' decision $\left\{\left.q_{k}\right|_{k \neq i}\right\}$ is the protect information to $\mathrm{ICD}_{i}$, and thus each ICD only can estimate the rivals' decision as $\widehat{q_{k}}$. From (7), $U_{i}$ is a continuous quadratic function of $q_{i}$ by assuming $\sum_{k \neq i} \hat{q_{k}}$ is fixed, and the second derivative of $U_{i}$ with respect to $q_{i}$ is $\frac{\partial^{2} U_{i}}{\partial q_{i}^{2}}=-2 \alpha$, where $\alpha>0$. As $\frac{\partial^{2} U_{i}}{\partial q_{i}^{2}}<0, U_{i}$ is a concave function of $q_{i}$. Therefore, by setting the first derivative equal to zero, the optimal output of $\mathrm{ICD}_{i}$ satisfies $q_{i}^{\text {opt }}=\left(p_{\max }-c_{i}-\alpha \sum_{k \neq i} \widehat{q}_{k}^{\text {opt }}\right) / 2 \alpha$. As $p$ is known, we can substitute (5) into the above equation, and obtain $q_{i}^{\text {opt }}$ expressed as (6). This finishes the proof of Theorem 1.

The next problem is to adjust the pricing strategy $p$ to maximize the utility of the EMS while guaranteeing its computational demand.

\section{B. Tier I: the optimal unit price strategy}

Under incomplete information, the EMS has a rough estimation on each ICDs' cost information. We consider that the unit cost of $\mathrm{ICD}_{i}$ is a variable following certain distributions. Let us denote $c_{i, n}$ as the unit cost of $\mathrm{ICD}_{i}$ with probability $\gamma_{i, n}$. The probability $\gamma_{i, n}$ satisfies $\sum_{n \in \mathcal{N}_{i}} \gamma_{i, n}=1$, where $n \in \mathcal{N}_{i} \triangleq\left\{1,2, \ldots, N_{i}\right\}$ indicates there are $N_{i}$ kinds of possible values for unit $\operatorname{cost} c_{i}$.

Theorem 2. Given the unit profit $P_{\mathrm{EMS}}$ and the computing workload $Q_{\mathrm{EMS}}$, the EMS can determine the optimal price strategy $p\left(Q_{\mathrm{ICD}}\right)$ by the the estimation of the unit cost of the ICDs, which can be given by

$$
\begin{aligned}
p^{\mathrm{opt}}\left(Q_{\mathrm{ICD}}\right)= & \left(\frac{I+1}{2} P_{\mathrm{EMS}}-\frac{I-1}{2 I} \sum_{i \in \mathcal{I}} \mathbb{E}_{n \in \mathcal{N}_{i}}\left[c_{i, n}\right]\right) \\
& -\frac{I P_{\mathrm{EMS}}-\sum_{i \in \mathcal{I}} \mathbb{E}_{n \in \mathcal{N}_{i}}\left[c_{i, n}\right]}{2 Q_{\mathrm{EMS}}} Q_{\mathrm{ICD}},
\end{aligned}
$$

where $\mathbb{E}_{n \in \mathcal{N}_{i}}\left[c_{i, n}\right]$ denotes the estimation of the unit cost of $\mathrm{ICD}_{i}$ and $Q_{\mathrm{ICD}}=\sum_{i \in \mathcal{I}} q_{i}$ denotes the enhanced computational capacity for emergency management on demand.

Proof: From (6) indicated in Theorem 1., the optimal amount of shared computing resources from $\mathrm{ICD}_{i}$ satisfies $q_{i, n}^{\mathrm{opt}}=\left(p-c_{i, n}\right) / \alpha$ when its actual unit cost is $c_{i, n}$. From the perspective of the EMS, it can determine the optimal strategy $q_{i}^{\text {opt }}=q_{i, n}^{\text {opt }}$ with the probability $\gamma_{i, n}$. Thus the expected amount of shared computing resources can be expressed as

$$
\begin{aligned}
\mathbb{E}\left[Q_{\mathrm{ICD}}\right] & =\sum_{i \in \mathcal{I}} \mathbb{E}_{n \in \mathcal{N}_{i}}\left[q_{i, n}^{\mathrm{opt}}\right]=\sum_{i \in \mathcal{I}} \sum_{n \in \mathcal{N}_{i}} \frac{p-c_{i, n}}{\alpha} \gamma_{i, n} \\
& =\frac{I p-\sum_{i \in \mathcal{I}} \sum_{n \in \mathcal{N}_{i}} c_{i, n} \gamma_{i, n}}{\alpha},
\end{aligned}
$$


where $\sum_{n \in \mathcal{N}_{i}} c_{i, n} \gamma_{i, n}$ is the expected unit cost of $\operatorname{ICD}_{i}$, which can be denoted as $\mathbb{E}_{n \in \mathcal{N}_{i}}\left[c_{i, n}\right]$.

Substituting (5) into (9), and the total expected amount of shared computing resources is $\mathbb{E}\left[Q_{\mathrm{ICD}}\right]=$ $\left(I p_{\max }-\sum_{i \in \mathcal{I}} \mathbb{E}_{n \in \mathcal{N}_{i}}\left[c_{i, n}\right]\right) / \alpha$. The expected utility of the EMS can be represented as (10) at the top of the next page by substituting the above equation into (1).

When $\alpha$ is fixed, $\mathbb{E}\left[U_{\mathrm{EMS}}\right]$ is a continuous quadratic function of $p_{\max }$. Similar to the proof of Theorem 1., we can obtain the optimal parameter $p_{\max }^{\mathrm{opt}}$ by setting the first derivative equal to zero as $p_{\max }^{\mathrm{opt}}=(I+1) P_{\mathrm{EMS}} / 2-$ $(I-1) \sum_{i \in \mathcal{I}} \mathbb{E}_{n \in \mathcal{N}_{i}}\left[c_{i, n}\right] / 2 I$.

From (10), we can obtain that $\mathbb{E}\left[U_{\mathrm{EMS}}\right]$ is a monotonically decreasing function with respect to $\alpha$. Substituting $p_{\max }^{\mathrm{opt}}$ back to $\mathbb{E}\left[Q_{\mathrm{ICD}}\right]$, and it can be rewritten as $\mathbb{E}\left[Q_{\mathrm{ICD}}\right]=$ $\left(I P_{\mathrm{EMS}}-\sum_{i \in \mathcal{I}} \mathbb{E}_{n \in \mathcal{N}_{i}}\left[c_{i, n}\right]\right) / 2 \alpha$. To satisfy the computation requirement from the EMS, the optimal parameter $\alpha^{\text {opt }}$ can be determined by replacing $\mathbb{E}\left[Q_{\mathrm{ICD}}\right]$ with $Q_{\mathrm{EMS}}$ as $\alpha^{\mathrm{opt}}=\left(I P_{\mathrm{EMS}}-\sum_{i \in \mathcal{I}} \mathbb{E}_{n \in \mathcal{N}_{i}}\left[c_{i, n}\right]\right) / 2 Q_{\mathrm{EMS}}$.

Finally, we substitute $p_{\max }^{\text {opt }}$ and $\alpha^{\text {opt }}$ into (5) and obtain the optimal unit price expressed as (8). This finishes the proof of Theorem 2.

In this way, the EMS and the ICDs can yield BNE $\left\{p^{\mathrm{opt}},\left\{q_{i, n}^{\mathrm{opt}} ; c_{i, n}\right\}_{n \in \mathcal{N}_{i}, i \in \mathcal{I}}\right\}$. Since there are $N_{i}$ kinds of possible values for $\mathrm{ICD}_{i}$, BNE is not unique. To address this problem, we design the N-IITG algorithm to iteratively get the unique near-optimal strategies of the EMS and the ICDs based on the IITG model. Next, we provide the details of the near-optimal algorithm.

\section{N-IITG Algorithm}

To address the uniqueness problem of incomplete information, we propose the N-IITG algorithm to yield the unique BNE of the EMS and the ICDs.

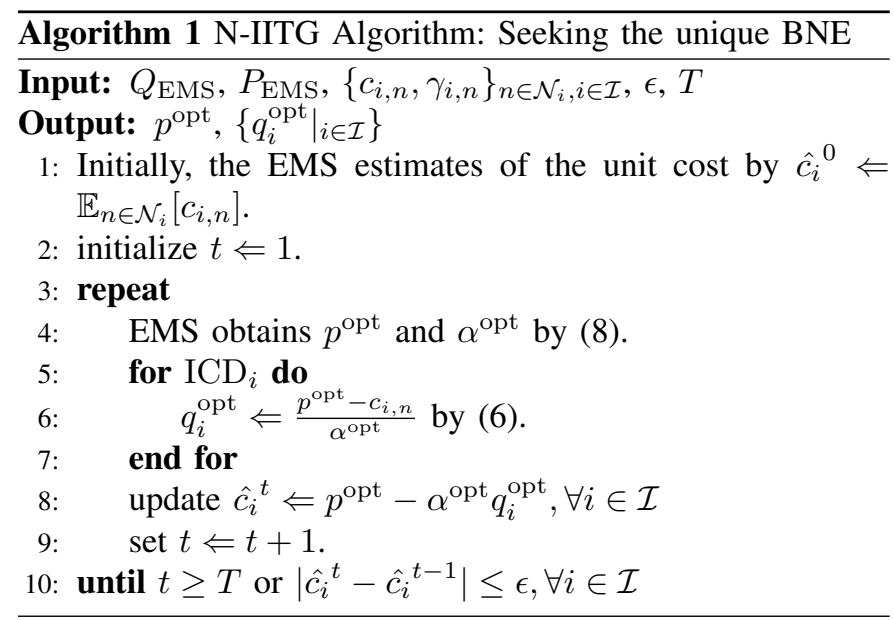

Different from the complete information scenario, the EMS and the ICDs need to find out the unique optimal strategies by iterations from Step 3 to Step 10. Under incomplete information model, the EMS only has the estimation of the unit cost of $\mathrm{ICD}_{i}$, and it initializes the estimated unit cost $\hat{c}_{i}$ in expectation and deduces the optimal unit price $p^{\text {opt }}$ by (8). Then, each ICD determines its particular optimal strategy $q_{i}^{\mathrm{opt}}$ by (6) based on the actual unit cost. In each iteration, the EMS can update the estimated unit cost $\hat{c_{i}}$ by the observed actions $q_{i}^{\text {opt }}$ from the ICDs. Then, the EMS and the ICDs recalculate their optimal strategies until the unit cost of each ICD converges to a stable value, or the number of iterations exceeds its maximum threshold. Finally, all participants achieve the unique BNE where no one will alter its strategy if the strategies of the others remain unchanged.

Next, we discuss the time complexity of the N-IITG algorithm. As shown in Algorithm 1, the time complexity of unit cost estimation is $\mathcal{O}[I]$. All participants can determine their strategies by the closed-form solution, i.e., $\mathcal{O}[1]$. Therefore, the overall time complexity is $\mathcal{O}[N I]$, where $N$ is the iteration count required to converge. This result demonstrates that the N-IITG algorithm can be finished in polynomial time.

\section{NumericAl Results}

In this section, we present numerical results to evaluate the performance of the N-IITG algorithm. Parameter settings are given first. The EMS's demand $Q_{\mathrm{EMS}}$ is set as to $50 \mathrm{MB}$ and $P_{\text {EMS }}$ to 20 per MB. We consider that there are 10 ICDs in proximity having idle computing resources, i.e., $I=10$. The unit cost $c_{i}$ for sharing computing resources is private information, i.e., each ICD knows well its actual unit cost $c_{i}$, while the EMS and the other ICDs only have a rough estimation based on the probability distribution, and then $c_{i}$ is estimated as $\hat{c_{i}}=\mathbb{E}_{n \in \mathcal{N}_{i}}\left[c_{i, n}\right]$.

Here, we take Bernoulli distribution for instance, and divide the unit cost into two types for simplicity, i.e., $c_{i} \triangleq\left\{c_{i}^{L}, c_{i}^{H}\right\}$, and the probability is $\Gamma_{i} \triangleq\left\{\gamma_{i}^{L}, \gamma_{i}^{H}\right\}$, where $\gamma_{i}^{L}+\gamma_{i}^{H}=1$. The low unit cost $c_{i}^{L}$ denotes low local computing intensity and transmission power, and $c_{i}^{H}$ is the exact opposite case. According to these settings, we assume that the unit cost $c_{i}$ is uniformly and randomly distributed over $[1,10](\$ / \mathrm{MB})$.

According to Theorem 2., the EMS determines its optimal unit price $p^{\text {opt }}$ based on the estimation of the unit cost, i.e.,

$$
\begin{aligned}
p^{\mathrm{opt}}\left(Q_{\mathrm{ICD}}\right)= & \left(\frac{I+1}{2} P_{\mathrm{EMS}}-\frac{I-1}{2 I} \sum_{i \in \mathcal{I}}\left(c_{i}^{L} \gamma_{i}^{L}+c_{i}^{H} \gamma_{i}^{H}\right)\right) \\
& -\frac{I P_{\mathrm{EMS}}-\sum_{i \in \mathcal{I}}\left(c_{i}^{L} \gamma_{i}^{L}+c_{i}^{H} \gamma_{i}^{H}\right)}{2 Q_{\mathrm{EMS}}} Q_{\mathrm{ICD}} .
\end{aligned}
$$

Then, according to Theorem 1., $\mathrm{ICD}_{i}$ determines its optimal amount of shared computing resources $q_{i}^{\text {opt }}$ according its actual unit cost, which can be given by

$$
q_{i}^{\mathrm{opt}}= \begin{cases}q_{i}^{L^{\mathrm{opt}}}=\frac{p^{\mathrm{opt}}-c_{i}^{L}}{\alpha}, & c_{i}=c_{i}^{L} \\ q_{i}^{H^{\mathrm{opt}}}=\frac{p^{\mathrm{opt}}-c_{i}^{H}}{\alpha}, & c_{i}=c_{i}^{H} .\end{cases}
$$

As shown in Fig. 2, we investigate the shared computing resources of each ICD under incomplete information and compare it with the complete information scenario. Here, the result of complete information can be derived by $c_{i}$ instead of $\hat{c}_{i}$. To analyze the difference of actual unit cost and estimated unit cost, we only set $c_{2}, c_{5}$ and $c_{8}$ as unknown parameters, which should be estimated by the expectation. As illustrated in Fig. 2 (a), the ICDs are willing to offload more workload 
$\mathbb{E}\left[U_{\mathrm{EMS}}\left(p_{\max }, \alpha\right)\right]=-\frac{I}{\alpha(I+1)^{2}}\left[p_{\max }-\left(\frac{I+1}{2} P_{\mathrm{EMS}}-\frac{I-1}{2 I} \sum_{i \in \mathcal{I}} \mathbb{E}_{n \in \mathcal{N}_{i}}\left[c_{i, n}\right]\right)\right]^{2}+\frac{1}{4 \alpha I}\left(I P_{\mathrm{EMS}}-\sum_{i \in \mathcal{I}} \mathbb{E}_{n \in \mathcal{N}_{i}}\left[c_{i, n}\right]\right)^{2}$.

than the EMS's prediction if actual unit cost is smaller than estimated unit cost, which makes the participants unable to reach equilibrium after one iteration. Next, we simulate the convergence iterations with our proposed N-IITG algorithm. The upper limit $\epsilon$ is set as $10^{-2}$. From Fig. 2 (b), the computing resources shared by each ICD converges to the value under complete information, and the total amount of the shared computing resources satisfy the EMS's computation requirement. This result indicates that the N-IITG algorithm can achieve a near-optimal performance of complete information under the condition of incomplete information.

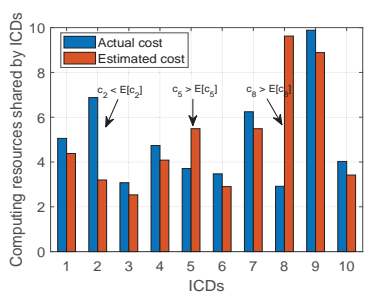

(a) One iterations

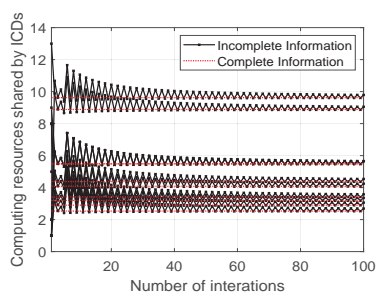

(b) Multiple iterations
Fig. 2. Shared computing resources versus $c_{i}$ and $\hat{c}_{i}$.

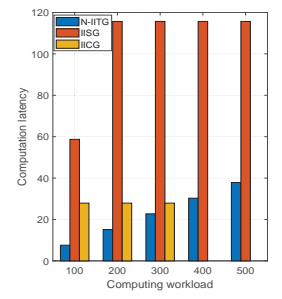

(a)

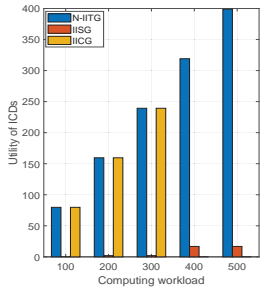

(b)

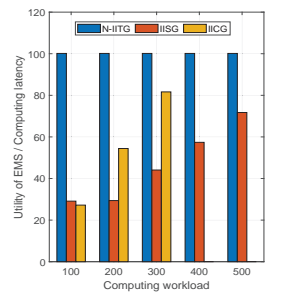

(c)
Fig. 3. Algorithm comparison versus computing workload.

In Fig. 3, we compare the performance of the N-IITG algorithm with two algorithms in terms of the computation latency and the participants' utilities, i.e., incomplete information based Stackelberg game algorithm (IISG) [9], and incomplete information based Cournot game algorithm (IICG) [10]. Considering that the unit cost is proportional to the square of processing rate [4], we define the computation latency as $\max \left\{q_{i} /\left.\operatorname{sqrt}\left(c_{i}\right)\right|_{i \in \mathcal{I}}\right\}$. Fig. 3(a) and 3(b) demonstrate that our proposed N-IITG algorithm can achieve a lower computation latency and a higher average utility of ICDs under different computing workloads ${ }^{1}$. Furthermore, apart from the utility, the computation latency is also important to the performance of the EMS in ECNs. Therefore, we compare the performance of the EMS by the ratio of the above two items, and the NIITG algorithm outperforms the other two algorithms as well.

\footnotetext{
${ }^{1}$ Since IICG cannot dynamically adjust its pricing strategy, the computing task fails to be processed when it increases to $400 \mathrm{MB}$ or above. Therefore, the computation latency of IICG is infinite, and the utilities are zero in Fig.3.
}

That is because our proposed N-IITG algorithm considers not only the interaction between the EMS and the ICDs but also the internal competitions among the ICDs. In this way, the EMS can dynamically adapt its pricing strategy to inspire the competitive ICDs for sharing sufficient computing resources, which contributes to relieve the computation pressure in ECNs. Each ICD involved in collaborative computing can maximize its utility by selecting the optimal computation workload.

\section{CONCLUSION}

In this letter, we proposed an IITG model to incentivize collaborative computing in ECNs, which jointly combined the Stackelberg game and the Cournot game. Depending on the given information of the EMS and the ICDs, we analyzed BNE of the EMS and the ICDs under incomplete information, and further designed the N-IITG algorithm that can iteratively convergent to the unique BNE. According to the simulation results, the proposed scheme achieved a significant increase in computational capacity while each participant obtained the optimal profit. For future work, it would be meaningful to study the collaborative computing problem in ECNs by combining cloud computing.

\section{REFERENCES}

[1] R. R. Murphy, "Emergency Informatics: Using Computing to Improve Disaster Management," in Computer, vol. 49, no. 5, pp. 19-27, May 2016.

[2] E. K. Markakis et al., "Efficient Next Generation Emergency Communications over Multi-Access Edge Computing," IEEE Commun. Mag., vol.55, no. 11, pp. 92-97, Nov. 2017.

[3] D. Xu et al., "A Survey of Opportunistic Offloading," in IEEE Commun. Surv. Tut., vol. 20, no. 3, pp. 2198-2236, thirdquarter 2018.

[4] Y. Mao, C. You, J. Zhang, K. Huang and K. B. Letaief, "A Survey on Mobile Edge Computing: The Communication Perspective," in IEEE Commun. Surv. Tut., vol. 19, no. 4, pp. 2322-2358, Fourthquarter 2017.

[5] N. C. Luong, P. Wang, D. Niyato, Y. Liang, Z. Han and F. Hou, "Applications of Economic and Pricing Models for Resource Management in 5G Wireless Networks: A Survey," in IEEE Commun. Surv. Tut., vol. 21, no. 4, pp. 3298-3339, Fourthquarter 2019.

[6] H. Fang, L. Xu and X. Wang, "Coordinated Multiple-relays based Physical-layer Security Improvement: A Single-leader Multiplefollowers Stackelberg Game Scheme," in IEEE Trans. Inf. Forensics Security, vol. 13, no. 1, pp. 197-209, Jan. 2018.

[7] F. Sun, F. Hou, H. Zhou, B. Liu, J. Chen and L. Gui, "Equilibriums in the Mobile-Virtual-Network-Operator-Oriented Data Offloading," in IEEE Trans. Veh. Technol., vol. 67, no. 2, pp. 1622-1634, Feb. 2018.

[8] L. Duan, T. Kubo, K. Sugiyama, J. Huang, T. Hasegawa and J. Walrand, "Motivating Smartphone Collaboration in Data Acquisition and Distributed Computing," in IEEE Trans. Mobile Comput., vol. 13, no. 10, pp. 2320-2333, Oct. 2014.

[9] J. Nie, J. Luo, Z. Xiong, D. Niyato and P. Wang, "A Stackelberg Game Approach Toward Socially-Aware Incentive Mechanisms for Mobile Crowdsensing," in IEEE Trans. Wireless Commun., vol. 18, no. 1, pp. 724-738, Jan. 2019.

[10] C. Li, J. Li, Y. Li and Z. Han, "Pricing Game with Complete or Incomplete Information about Spectrum Inventories for Mobile Virtual Network Operators," in IEEE Trans. Veh. Technol., vol. 68, no. 11, pp. 11118-11131, Nov. 2019. 